Gut, 1978, 19, 587-592

\title{
Sialic acids of human large bowel mucosa: o-acylated variants in normal and malignant states
}

\author{
C. M. ROGERS, K. B. COOKE, AND M. I. FILIPE \\ From the Departments of Histopathology and Chemical Pathology, Westminster Medical School, London
}

SUMMARY Five sialic acid variants have been demonstrated in normal, 'transitional', and tumour extracts of human large bowel mucosa by thin-layer chromatography. Two-dimensional thin-layer chromatography, with intermediate alkaline hydrolysis, confirmed that the variants were $\mathrm{N}$-acetyl neuraminic acids with additional $\mathrm{O}$-acylation. Cases studied so far show changes in the proportions of these variants in malignancy.

\begin{abstract}
Abnormalities of the mucosa adjacent to human gastrointestinal cancers are well established (Nairn, 1969; Lipkin et al., 1970), changes in the mucosubstances of these areas being especially well demonstrated in the large bowel (Filipe, 1969, 1972; Filipe and Branfoot, 1976). Increases in mucosubstances staining as sialomucins in tumouradjacent mucosa ('transitional') as compared with the normal-that is, mucosa remote from the tumour-have been confirmed biochemically by sialic acid estimations (Filipe and Cooke, 1970). The sialic acid heterogeneity observed by Filipe and Cooke (1974) in normal and transitional mucosa, was confirmed for normal mucosa by Reid and co-workers (1975). Both groups used the neuraminidase of Vibrio cholerae to release sialic acids other than 4-0-acyl variants (which are resistant to the enzyme) and the latter group combined this with acid hydrolysis before and after ester removal by saponification. Many sialic acid variants have been described in animal tissues (Gottschalk, 1963; Schauer, 1973), but the analogous heterogeneity in human large intestinal mucosa has not hitherto been demonstrated directly.
\end{abstract}

\section{Methods}

One to two grams of tumour, normal, and transitional mucosa, scraped from surgical specimens of the left colon and rectum from human large

${ }^{1}$ The temperature rise after one minute of sonication under these conditions was less than $10^{\circ} \mathrm{C}$; sonication of BSM in solution for up to $1 \frac{1}{\mathrm{z}}$ minutes resulted in no detectable degradation of sialic acids.

Received for publication 9 December 1977 intestine resected for carcinoma were treated as shown in Fig. 1. Sialic acids were extracted from bovine salivary mucin (BSM) in the same way, for comparison purposes. Tissues, stored at $-70^{\circ} \mathrm{C}$ until use, were minced while thawing and sonicated as $^{1}$ standard procedure for one minute using an MSE Ultrasonic Disintegrator (the 60 watt generator being tuned maximally to approximately 20000 cycles/s). They were then homogenised over an ice bath with a MSE homogenizer run at maximum speed (approximately $14000 \mathrm{rpm}$ ) for one minute. Hydrolytic release of sialic acids was effected by adjusting the $\mathrm{pH}$ of the dialysed homogenates to 1.9-2.0 with formic acid and incubating them for 60 minutes at $70^{\circ} \mathrm{C}$ (Buscher et al., 1974), and was followed by dialysis (with stirring) against 0.51 of distilled water for $10-20$ hours at $2^{\circ} \mathrm{C}$. The sialic acid-containing diffusates were concentrated on a rotary evaporator at $30^{\circ} \mathrm{C}$ under reduced pressure. The sialic acids were desalted by ion exchange chromatography using coupled columns $(0.7 \times 4 \mathrm{~cm})$, the upper containing Bio-Rad AG50W-X8 (H+ form) and the lower, Bio-Rad AG2-X8 (HCOO - form). Samples were applied to the upper columns, eluted with water, and the upper colums removed. Non-sialyl material was washed from the lower columns with 0.01-N formic acid. Sialic acid-containing fractions were eluted in 2-N formic acid. Thin-layer chromatography (TLC) was performed on pre-coated polyethylene-backed cellulose plates $(20 \mathrm{~cm} \times 20 \mathrm{~cm} \times 0.1 \mathrm{~mm}$, catalogue no. 5577, from E. Merck, Darmstadt). The plates were prerun in $0 \cdot 1-\mathrm{N}$ hydrochloric acid and developed in the same direction, in butan-1-ol/ propan-1-ol/0 1-N hydrochloric acid ( $1: 2: 1$ volumes) (Buscher et al., 1974); 15-20 $\mu$ l of each sample was 


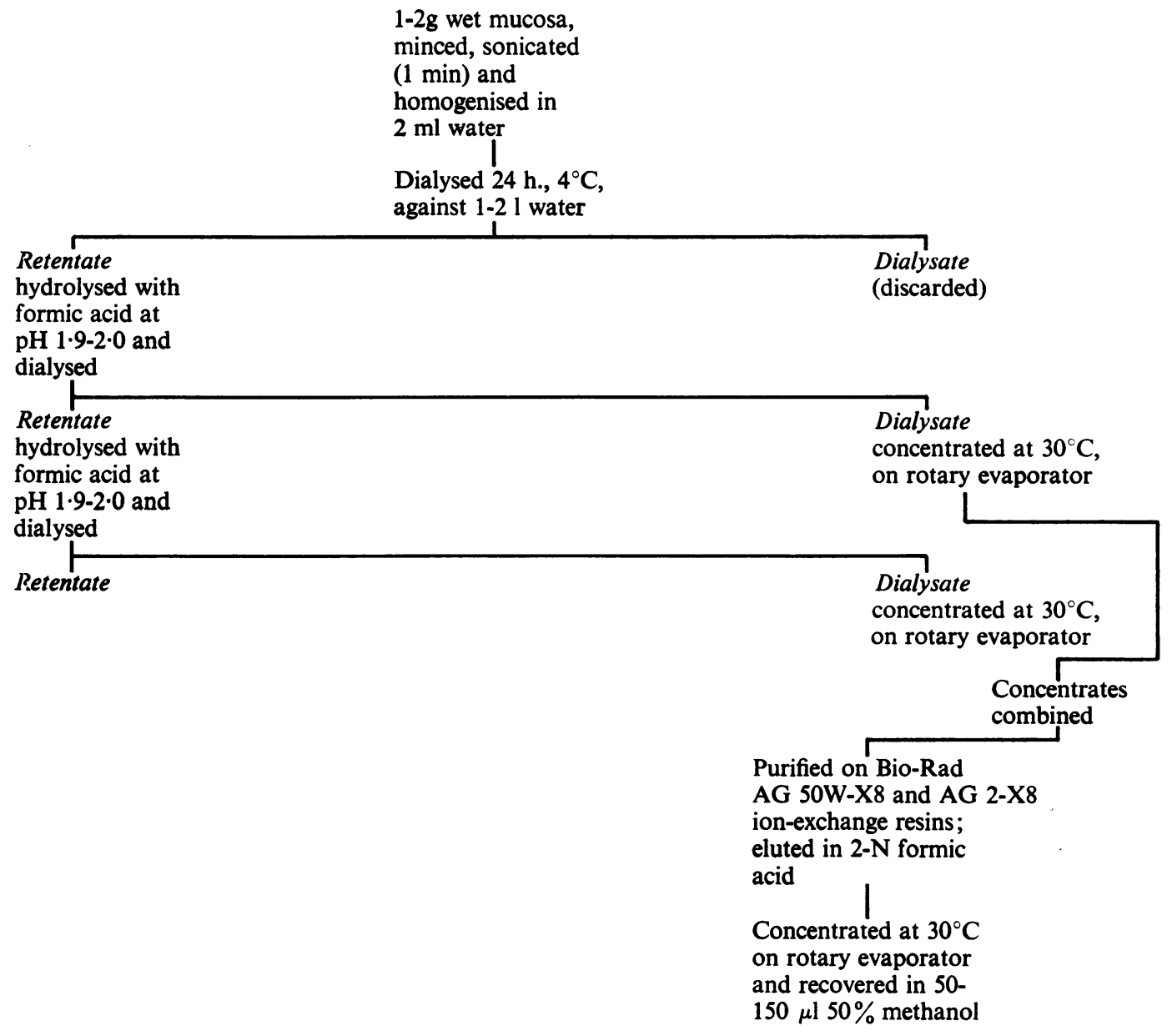

Fig. 1 Preparation of sialic acids for TLC.

applied as $1-\mathrm{cm}$ streaks along the origin line. $\mathrm{N}$-acetyl neuraminic acid (Sigma Chemical Co.) and sialic acids from BSM (a gift from Dr A. P. Corfield) were used as reference compounds. Runs were $10-18 \mathrm{~cm}$.

All development solvents were prepared fresh on the day of use. Two-dimensional thin-layer chromatography was performed on Silicagel 60, HPTLC plates (Merck), developed in propan-1-ol/ water ( $7: 3$ volumes). To confirm the presence of 0-acyl groups, alkaline hydrolysis was carried out in ammonia vapour between runs. After development and drying in warm air, the sialic acids were stained with the orcinol spray (Bohm et al., 1954). BSM-sialic acids, applied before development in the second direction, were used as reference compounds.

\section{Results}

Separation and identification of sialic acids was performed on a total of five cases of large bowel cancer.

\section{UNIDIMENSIONAL THIN-LAYER CHROMA-} TOGRAPHY OF SIALIC ACIDS

Thin-layer chromatograms of two of the five cases studied are shown in Fig. 2. The mean $R f$ values of the separated bands are listed in the Table together with those from BSM, for comparison. The $\mathbf{R f}$ values for all BSM sialic acids variants except $\mathrm{N}$-glycollyl neuraminic acid (band 6) agreed to within $\pm 6 \%$ ( $-18 \%$ for band 6 ), with those obtained by Professor R. Schauer's laboratory using the same chromatographic conditions (A. P. Corfield, per- 

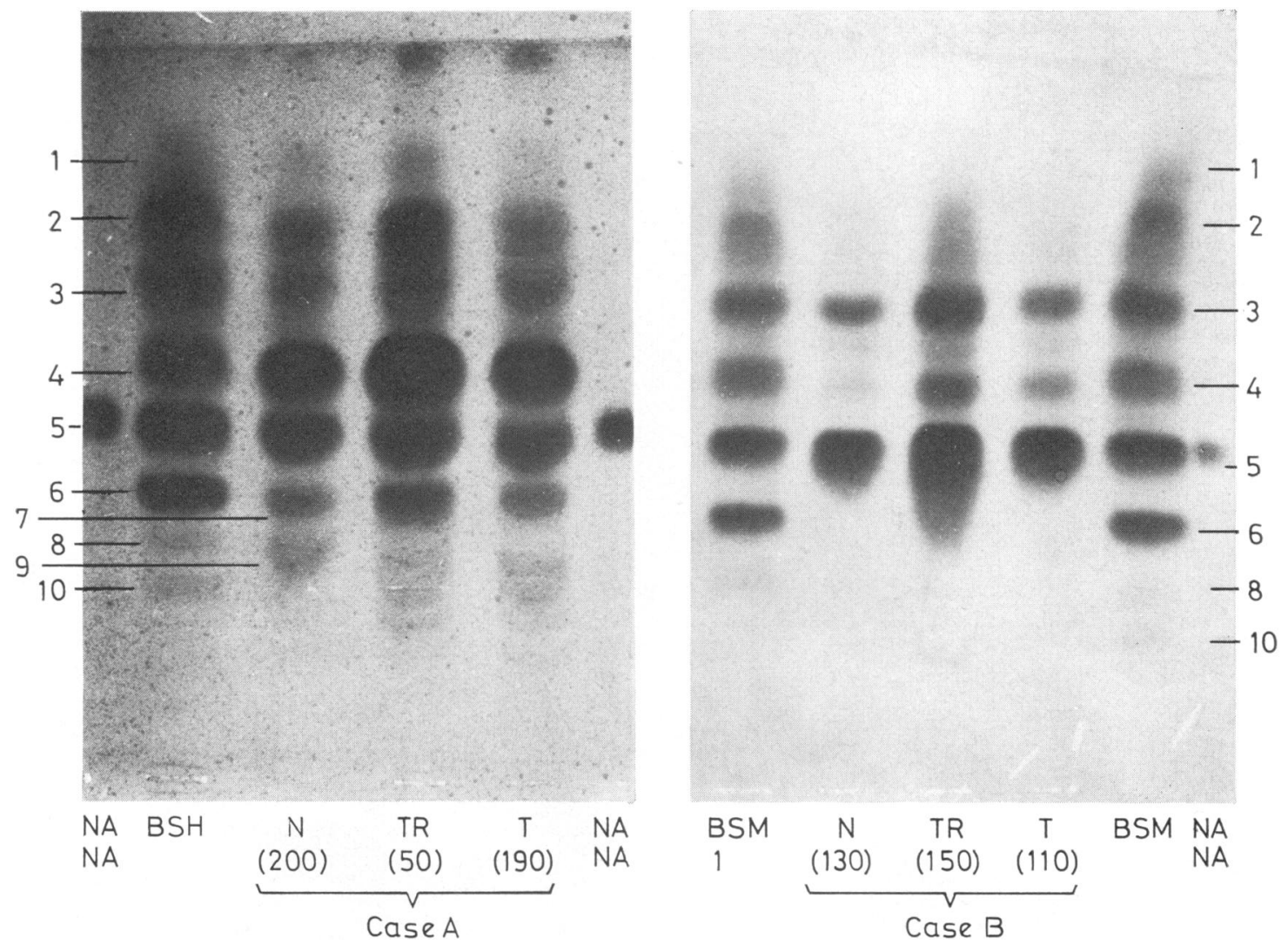

Fig. 2 Thin-layer chromatography of sialic acids from two cases of large bowel carcinoma. (Wet weights of human mucosa in milligrams are given in parentheses; $N$ : normal mucosa; TR : 'transitional' mucosa; $T$ : tumour; NANA: $N$-acetyl neuraminic acid; BSM : bovine salivary muc·n).

Table Mean $R f$ values of human, bovine, and synthetic sialic acids stained with orcinol

\begin{tabular}{|c|c|c|c|c|c|c|c|c|c|c|}
\hline \multirow[t]{2}{*}{ Orcinol positive bands } & \multicolumn{5}{|l|}{ Case } & \multirow[t]{2}{*}{ Mean } & \multirow[t]{2}{*}{ $\pm S D$} & \multirow[t]{2}{*}{ BSM-bands } & \multirow[t]{2}{*}{$N A N A^{*}$} & \multirow[t]{2}{*}{ $\pm S D$} \\
\hline & $\boldsymbol{A}$ & $\boldsymbol{B}$ & $C$ & $D$ & $E$ & & & & & \\
\hline \multicolumn{11}{|c|}{$\mathrm{N}$-acetyl neuraminic acids } \\
\hline 1 & 0.83 & 0.83 & - & 0.83 & 0.81 & 0.83 & 0.01 & 0.84 & - & 0.01 \\
\hline 2 & 0.76 & 0.75 & 0.72 & 0.76 & 0.70 & $0 \cdot 73$ & 0.02 & 0.74 & - & 0.01 \\
\hline 3 & 0.65 & 0.65 & 0.63 & 0.68 & 0.63 & 0.65 & 0.02 & 0.65 & - & 0.02 \\
\hline 4 & 0.55 & 0.55 & 0.53 & 0.58 & 0.52 & 0.53 & 0.02 & 0.55 & - & 0.03 \\
\hline 5 & 0.45 & 0.46 & 0.45 & 0.49 & 0.44 & 0.46 & 0.02 & 0.45 & 0.46 & 0.03 \\
\hline \multicolumn{11}{|l|}{ Other chromogens } \\
\hline 6 & - & - & - & - & - & - & - & $0 \cdot 36$ & - & 0.03 \\
\hline 7 & $0 \cdot 37$ & - & 0.35 & 0.42 & $0 \cdot 32$ & $0 \cdot 36$ & 0.04 & - & - & - \\
\hline 8 & - & - & - & - & - & - & - & $0 \cdot 30$ & - & 0.04 \\
\hline 9 & $0 \cdot 29$ & - & 0.28 & 0.35 & 0.24 & 0.29 & 0.04 & - & - & - \\
\hline 10 & - & - & - & - & - & - & - & 0.24 & - & 0.04 \\
\hline
\end{tabular}

*NANA : N-acetyl neuraminic acid.

sonal communication), so that a tentative identification for the human sialic acids can be made from Schauer's data for BSM.

Fig. 3 shows the effect of strong alkali on the migrations of human and bovine sialic acids on silica gel: exposure of human sialic acids to am- monia vapour after development in the first direction results in compounds with the mobility of $\mathrm{N}$-acetyl neuraminic acid when developed in the second direction. Similar treatment of BSM-sialic acids is seen to produce components with the mobility of either $\mathrm{N}$-acetyl or $\mathrm{N}$-glycollyl neuraminic acid. 


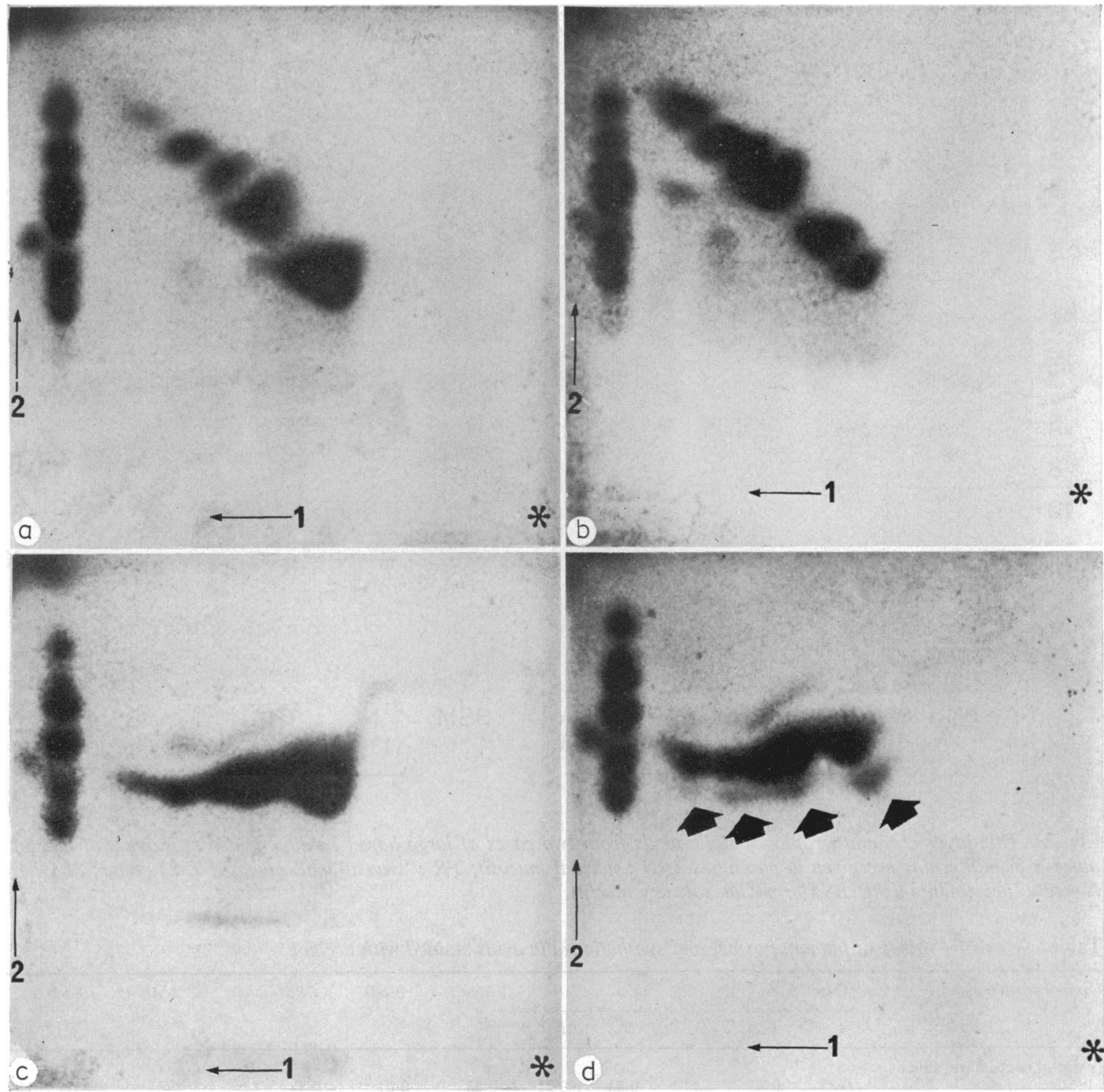

Fig. 3 Two-dimensional TLC of sialic acids from normal human mucosa $(a, c)$, and BSM $(b, d):(a)$ and $(b)$, without intermediate saponification; $(c)$ and $(d)$, with intermediate saponification (origins marked with an adjacent asterisk, directions of development are indicated by arrows).

\section{Discussion}

The five sialic acid bands seen in cases $\mathbf{A}$ and $\mathbf{B}$ (Fig. 2) were present in all samples prepared and analysed with minimal prior storage.

Sialic acids from BSM are identified on the basis of their Rf values:

Band 6: N-glycollyl neuraminic acid

Band 5: N-acetyl neuraminic acid

Band 4: $\mathrm{N}$-acyl, $\mathrm{O}$-acyl neuraminic acids $\left.\begin{array}{l}\text { Band } 3 \\ \text { Band } 2\end{array}\right\}: \mathrm{N}$-acyl, di-O-acyl neuraminic acids

Band 1: $\mathrm{N}$-acyl, tri-O-acyl neuraminic acids

Bands from human mucosa, labelled 1-5, are analogous to bands 1-5 from BSM samples, their Rf values being in agreement with those of the BSM bands:

Band 5: N-acetyl neuraminic acid ( $R f$ is the same as that of the synthetic $\mathrm{N}$-acetyl neuraminic acid reference sample) 
Band 4: $\mathrm{N}$-acetyl, O-acyl neuraminic acids

$\left.\begin{array}{l}\text { Band } 3 \\ \text { Band } 2\end{array}\right\}: \mathrm{N}$-acetyl di-O-acyl neuraminic acids

Band 1: N-acetyl, tri-O-acyl neuraminic acids

An additional band was observed in all samples analysed from case $B$, running between bands 3 and 4 (Fig. 2). This has not been seen in other cases or in BSM samples and, while having a mobility between those of the monosubstituted and the slowest disubstituted $\mathrm{N}$-acyl neuraminic acids, its $R f$ value (0.60) was not characteristic of any known sialic acid. This component is at present being investigated in collaboration with Professor Schauer's group.

With two-dimensional TLC on silica gel (Fig. 3) the migration of human sialic acid variants from normal mucosa as $\mathrm{N}$-acetyl neuraminic acid after alkaline hydrolysis (saponification) confirms their identity as $\mathrm{O}$-acylated $\mathrm{N}$-acetyl neuraminic acids. $\mathrm{O}$-acylated $\mathrm{N}$-glycollyl neuraminic acids would run, after saponification, in the same position as the $\mathrm{N}$-glycollyl neuraminic acid spot from the BSM reference sample, in the second direction; this, in fact, is seen in BSM (arrowed, Fig. 3d) but not in the sample of human origin. By analogy with the behaviour of BSM-sialic acids on storage (A. P. Corfield, personal communication) we identify the bands seen behind the fifth band in case $A$ as chromogens resulting from sialic acid degradation under storage conditions: they originate from substituted $\mathrm{N}$-acetyl neuraminic acids and are comparable with similar bands arising from substituted N-glycollyl neuraminic acids in stored BSM samples. In two cases (cases $C$ and $E$ ) showing considerable de-O-acylation of human sialic acids, these bands were present in greater amounts. Furthermore, they were absent from the profiles of case B. N-glycollyl neuraminic acid, running in a similar position to one of these bands, has been reported in humans in one instance (Gold and Miller, 1974) and we cannot yet exclude the possibility that $\mathrm{N}$-glycollyl neuraminic acids occur as genetically controlled variants.

The amounts of wet mucosa represented by the sialic acid extracts are given for the cases shown in Fig. 2, the relationship between mucosal net weight and protein content having been shown previously not to vary significantly (Filipe and Cooke, 1974). Our thin-layer chromatograms thus usually showed greater amounts of all types of sialic acids from transitional mucosa than from normal or tumour samples. This is in agreement with consistent increases observed in our laboratory in sialic acids from transitional mucosa as compared with the normal (Filipe and Cooke, 1974), but the increase had not previously been shown to reflect increases in the levels of all types of sialic acids.

Normal, transitional, and tumour samples each showed differences in the relative proportions of sialic acid variants within a sample; the most striking of which was a decrease in the amounts of more heavily substituted sialic acids (bands 1 and 2) in tumour material, as compared with the normal. This decrease is not accompanied by any increase in chromogens (see case B) and is therefore not due to increased necrosis within the tumour area. Transitional mucosa showed relative increases in bands 1 and 2 . In addition, case $B$ shows a greater difference in the relative quantities of band 4 between normal and tumour samples than the individual variation between cases shown by cases $A$ and $B$ in Fig. 2.

Filipe and Cooke (1974) suggested that the production of less complex sialic acids in association with malignancy might represent reversion to a more primitive state. Our results, indicating relative deficiencies in complex sialic acids from tumour tissue, support this hypothesis. However, increases in complex sialic acids from transitional mucosa have not previously been observed. The demonstration of at least five sialic acid types in human large bowel mucosa, not previously shown directly, confirms previous results by us and other groups that indicate the heterogeneity of sialic acid. Application of the methods described here may be of considerable use in the investigation and clinical surveillance of certain pathological conditions.

We gratefully acknowledge the advice and help of Professor R. Schauer and Dr A. P. Corfield, of the Ruhr-Universität, Bochum. C.M.R. was in receipt of grants from the Cancer Research Campaign and the Westminster Hospital Special Trustees.

\section{References}

Bohm, P., Dauber, S., and Baumeister, L. (1954). Über Neuraminsäure, ihr Vorkommen und ihre Bestimmung im serum. Klinische Wochenschrift, 32, 289-292.

Buscher, H. P., Casals-Stenzel, J., and Schauer, R. (1974). New sialic acids. European Journal of Biochemistry, 50, 71-82.

Filipe, M. I. (1969). The value of histochemical reactions for muco-substances in the diagnosis of certain pathological conditions of the colon and rectum. Gut, 10, 577-586.

Filipe, M. I. (1972). The value of a study of the mucosubstances in rectal biopsies from patients with carcinoma of the rectum and lower sigmoid in the diagnosis of premalignant mucosa. Journal of Clinical Pathology, 25, 123-128.

Filipe, M. I., and Branfoot, A. C. (1976). Mucin histochemistry of the colon. Current Topics in Pathology, 63, 143-178. 
Filipe, M. I., and Cooke, K. B. (1970). Changes in epithelial mucosubstances in mucosa immediately adjacent to carcinoma of the large intestine: a histochemical, autoradiographic and biochemical study. Abstract, Proceedings of the 4th World Congress of Gastroenterology, p. 281. Copenhagen.

Filipe, M. I., and Cooke, K. B. (1974). Changes in composition of mucin in the mucosa adjacent to carcinoma of the colon as compared with the normal: a biochemical investigation. Journal of Clinical Pathology, 27, 315-318.

Gold, D. V., and Miller, F. (1974). Characteristics of human colonic muco-protein antigen. Immunochemistry, 2, 369-375.

Gottschalk, A. (1963). 'Conjugated proteins' section B, glycoproteins and glycopeptides. Comprehensive Bio- chemistry, 8, 17-37.

Lipkin, M., Deschner, E., and Troncale, F. (1970). Cell differentiation and the development of colonic neoplasms. Gastroenterology, 59, 303-307.

Nairn, R. C. (1969). Fluorescent Protein Tracing, chapter 9 (Tissue antigens and antibodies), p. 248. Edited by R. C. Nairn. Livingstone: London.

Reid, P. E., Culling, C. F. A., Tsang, W.-C., Clay, M. G., Ramey, C. W., and Dunn, W. L. (1975). The demonstration of O-acetylated sialic acids in colonic epithelial glycoproteins. Canadian Journal of Biochemistry, 53, 388-391.

Schauer, R. (1973). Chemistry and biology of the acylneuraminic acids. Angewandte Chemie (International). 12, 127-138. 